\title{
Diagnosis and laparoscopic treatment of an unusual case of advanced extragenital endometriosis
}

\author{
J. English • K. Baig • T. Liston • G. Hudelist
}

Received: 28 February 2007 / Accepted: 14 June 2007 /Published online: 15 August 2007

(C) Springer-Verlag 2007

\begin{abstract}
Endometriosis is considered to be a benign gynaecological disorder, although several pathophysiological aspects of endometrial lesions resemble the behaviour of malignant tissue: similar to carcinomas, endometriotic cells are able to invade and destroy surrounding anatomical structures. Although the medical treatment of endometriotic lesions, including the use of $\mathrm{GnRH}$ analogues or gestagens, show temporary effectiveness and have been reported to cause a regression of disease, they rarely provide long-term relief of symptoms in advanced stages of endometriosis involving extragenital organs, such as the rectum or the urinary system. We here describe the diagnosis and minimally invasive surgical treatment of an unusually advanced case of endometriosis involving the rectosigmoid, the urinary bladder and the ureter, leading to secondary hydronephrosis and loss of renal function.
\end{abstract}

\footnotetext{
J. English $\cdot$ G. Hudelist $(\bowtie)$

Department of Obstetrics and Gynaecology,

Worthing and Southlands Hospital,

Lyndhurst Road,

West Sussex BN11 2DH, UK

e-mail: gernot_hudelist@yahoo.de

J. English

e-mail: james.english@wash.nhs.uk

K. Baig

Department of Surgery, Worthing and Southlands Hospital, Worthing, UK

T. Liston

Department of Urology, Worthing and Southlands Hospital, Worthing, UK

G. Hudelist

Department of Obstetrics and Gynaecology, LKH Villach, Villach, Austria
}

\begin{abstract}
Keywords Endometriosis $\cdot$ Laparoscopy $\cdot$ Surgery
\end{abstract}
\section{Introduction}

Endometriosis is characterised by the presence of endometrial tissue outside the uterine cavity and affects about $10 \%$ of the premenopausal female population [1]. The disease, albeit considered to be a "benign" one, can resemble the biological behaviour of malignant tumours: endometriotic cells attach to and invade surrounding tissues, thereby, causing symptoms such as pelvic pain, dypareunia, dyschezia or infertility. Over the past few decades, a number of treatment strategies have been evaluated, although none have been proven to be entirely effective. Various medical treatments for endometriosis, such as non-steroidal antirheumatics, GnRH analogues or contraceptive pills do not appear to exhibit significant differences in their effectiveness and only last as long as the patients remain on their medication $[2,3]$. In addition, antihormonal preparations such as GnRH analogues and/ or analgetic medications should not be prescribed on a long-term basis due to severe side-effects, including hot flushes, decrease of libido and bone mineral density, depression or impairment of renal function. Although radical surgical treatment for endometriosis remains an issue of constant debate, a number of studies, including randomised controlled trials, strongly suggest that excisional radical surgery is, indeed, highly effective in the treatment for endometriosis and warrants long-term curative effects regarding pelvic pain and subfertility [3-9]. However, some patients exhibit extensive involvement of extragenital tissues, such as the sigmoid and rectum, ureter and bladder, which confers a technical challenge for the surgeon, especially in a fertilitypreserving treatment approach. We here describe the preoperative diagnosis and fertility-preserving laparoscopic treatment of an unusual case of extraordinary extensive extragenital 


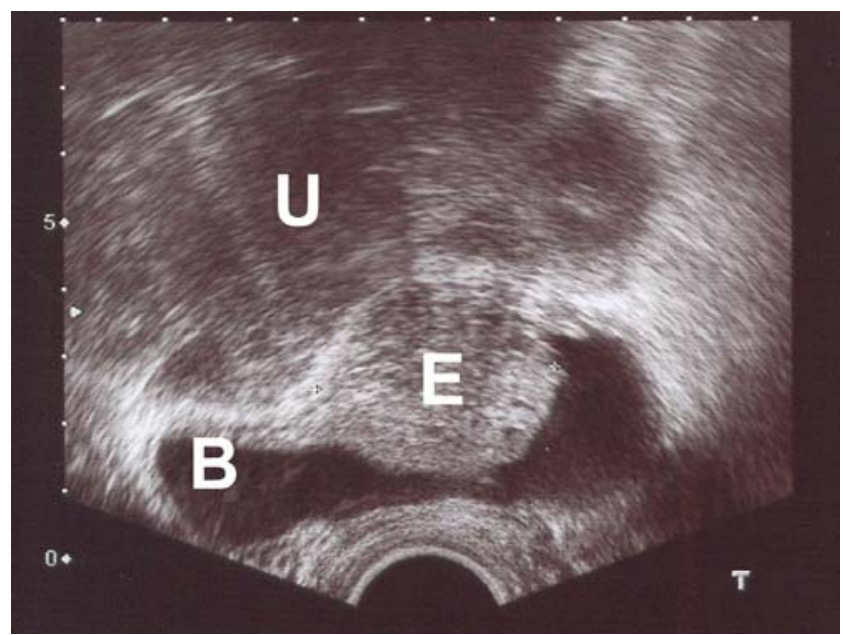

Fig. 1 Transvaginal ultrasound (transverse section) of the urinary bladder, showing a round-shaped hypoechogenic mass involving the posterior wall of the bladder, protruding into the bladder cavity. $U=$ uterus; $B=$ bladder; $E=$ endometriotic nodule

disease involving the urinary bladder, the rectum and sigmoid colon and the ureter.

\section{Case report}

A 26-year old (gravida 0 para 0 ) female of Caucasian origin presented with a long-standing history of dysmenorrhea, pelvic pain, dyspareunia, severe dyschezia and primary subfertility at our department. In addition, haematuria and dysuria with intermittent episodes of frequency had developed within the past 3 months. Her medical history did not reveal any abnormalities and she was treated for common menstrual pain with non-steroidal antirheumatics and opioids over the recent few years. At presentation in

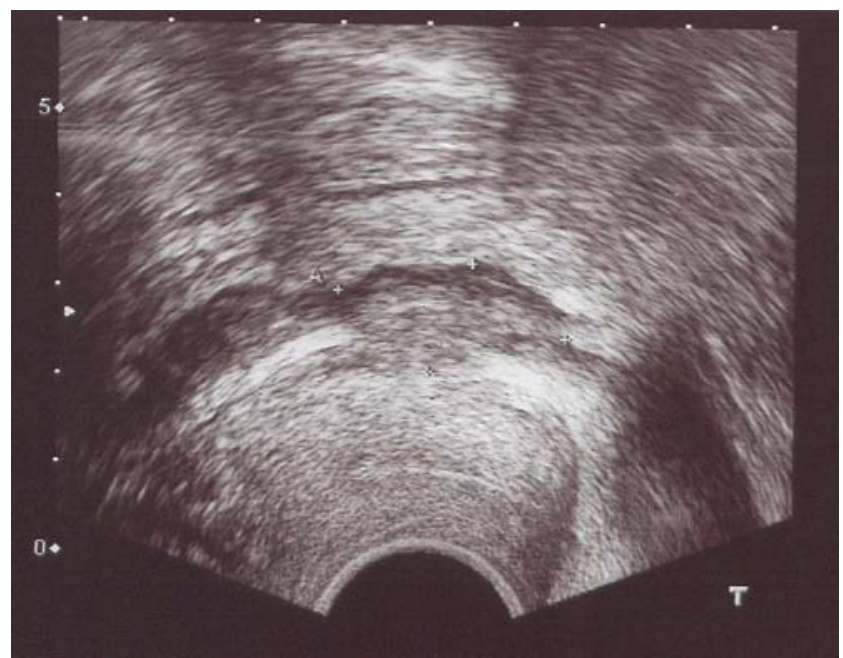

Fig. 2 Transvaginal ultrasound of the rectovaginal space, showing a hypoechogenic structure with a hyperechogenic halo distorting the hypo- and hyperechogenic layer of the anterior rectal wall, thereby, suggesting infiltration of the rectal muscularis

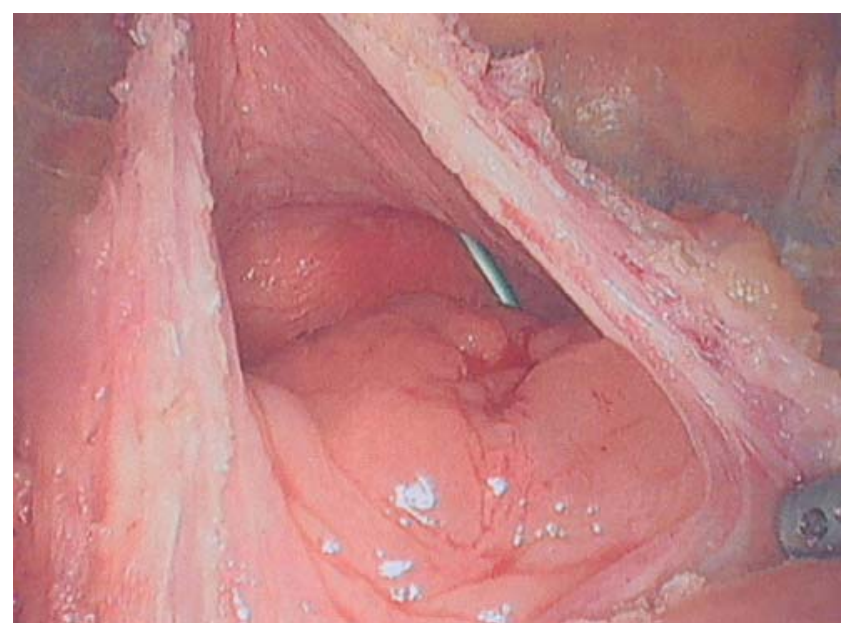

Fig. 3 Laparoscopic incision of the urinary bladder, revealing a nodular, livid structure infiltrating the posterior wall of the bladder

September 2006, urinalysis revealed microhaematuria and moderate leukocyturia, lacking the significant growth of organisms on urine culture. However, an ultrasound scan of her kidneys exhibited signs of hydronephrosis of her left kidney that was reconfirmed as complete hydronephrotic failure by a Mercapto Acetyl Tri Glycine (MAG3) scan exhibiting $11 \%$ residual renal function. On clinical examination, a fixed and retroverted uterus and a hard nodular mass of about $1.5 \mathrm{~cm}$ in diameter originating from the upper third of the rectovaginal space (RVS) with queried infiltration of the anterior rectal wall were palpated.

A transvaginal ultrasound scan of her pelvis finally revealed a $3.7 \times 4 \times 3 \mathrm{~cm}$ hyperechogenic, round-shaped mass originating from the posterior wall of the urinary bladder, protruding into the bladder cavity (Fig. 1). In addition, a $3 \times 1.3 \times 2 \mathrm{~cm}$ hypoechogenic mass with a hyperechogenic halo was located in the upper third of the RVS, distorting the hypo- and hyperechogenic layer of the

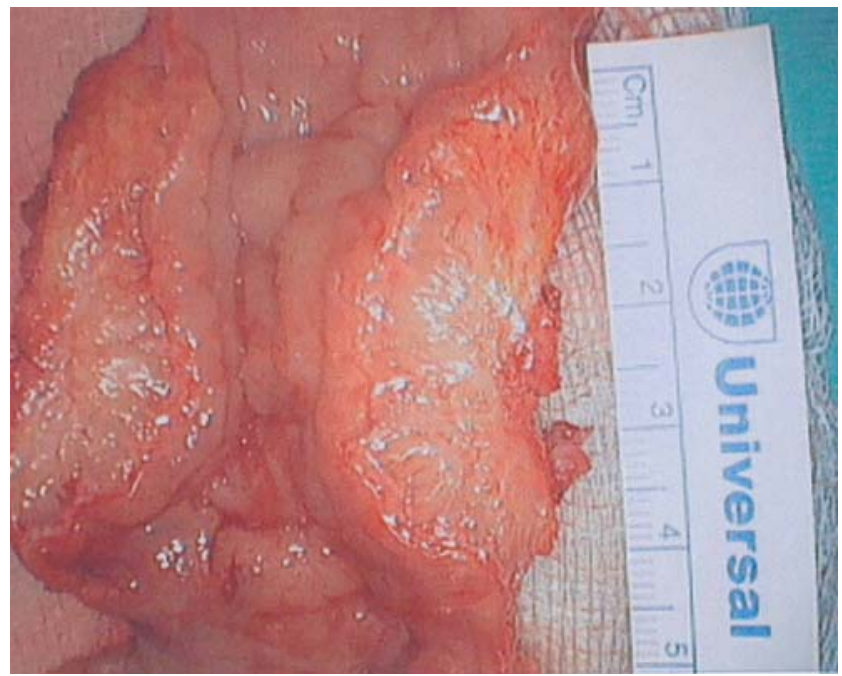

Fig. 4 Rectum with luminal obstruction caused by endometriotic infiltration of the anterior rectal wall 
anterior rectal wall, suggesting infiltration of the rectal muscularis (Fig. 2). A similar structure of size $1.2 \times 1 \times$ $1.3 \mathrm{~cm}$ was identified at the level of the uterine fundus, again suggesting infiltration of the rectosigmoid. Furthermore, a $2 \times 1.5 \times 3 \mathrm{~cm}$ round-shaped isoechogenic mass was identified on the right ovary, suggesting endometrioma of the ovary. Flexible cystoscopy for further evaluation of her haematuria revealed a nodular, livid structure infiltrating the posterior wall of the bladder. Finally, a diagnostic laparoscopy performed 3 months prior to the surgical procedure revealed a "frozen pelvis," strongly suggestive of endometriosis of the bowel. Based on these findings, the patient was admitted for laparoscopic radical resection of endometriosis and the left nephrectomy.

At operation, incision of the bladder showed macroscopic features of an endometriotic tumour originating from the posterior bladder wall (Fig. 3). In addition, dissection of the pouch of Douglas revealed extensive endometriosis of the RVS and anterior rectal wall. An isolated endometriotic nodule of the rectal wall was identified $6 \mathrm{~cm}$ cranial to the lower infiltration site. Following salpingo-ovariolysis, an endometrioma of the right ovary was drained and excised. The patient finally underwent anterior segmental resection of the rectal wall with end-to-end anastomosis $(31 \mathrm{~cm}$ Endogia $^{\circledR}$ device) (Fig. 4). Partial cystectomy involving resection of the posterior wall of the urinary bladder and suturing of the resection margins, as well as the left laparoscopic nephrectomy, was carried out. Histological analysis of paraffin-embedded tissue confirmed endometriosis of the right ovary, rectal muscularis (muscularis propria and submucosal layer) and the urinary bladder. Patho-histological analysis of the left kidney revealed a $6 \times$ $4 \times 3.5-\mathrm{cm}$ specimen and a $25-\mathrm{cm}-$ long ureter. Histological analysis demonstrated generalised renal atrophy due to hydronephrosis, as well as extrinsic and intrinsic endometriosis of the ureter. Her postoperative course was uncomplicated and the urinary catheter was removed on day 8 after the operation without complication. The patient was finally discharged following a control cystogram to exclude leakage. At follow-up four weeks postoperatively, the patient was complaining of moderate symptoms of urge incontinence but was otherwise doing well. A bladder scan showed minimal residual volume.

\section{Discussion}

The most frequent localisations of endometriotic lesions are the uterosacral ligaments, the pelvic peritoneum and the ovaries [10]. In a subgroup of patients, endometriotic growth is deeply infiltrating, i.e. the presence of endometriotic tissue more than $5 \mathrm{~mm}$ under the peritoneum [11]. Deep infiltrating endometriosis, which is commonly involving the RVS and the rectum, can be diagnosed in up to $10 \%$ of all patients with endometriosis [11]. However, endometriotic involvement of the urinary system is rather rare and has been reported to occur in only $1-2 \%$ of all cases of pelvic endometriosis [12]. Urinary endometriosis commonly involves the urinary bladder and/or the ureter. However, due to its rarity and asymptomatic course in the early stages of the disease, final diagnosis can be delayed [13]. In the case presented, endometriotic tissue not only involved the rectosigmoid but also caused considerable distortion of the urinary bladder due to extensive involvement of the posterior wall. In addition, ureteral obstruction finally led to hydronephrosis with a subsequent long-standing impairment and, finally, a loss of renal function. Although there is evidence of temporary regression of endometriosis under hormonal treatment, such as GnRH analogues or gestagens, we suggest that, in cases of extensive involvement of extragenital tissues, such as the bowel or the urinary tract, surgical therapy is mandatory and should especially be considered in patients with subfertility.

\section{References}

1. Gao X, Outley J, Botteman M, Spalding J, Simon JA, Pashos CL (2006) Economic burden of endometriosis. Fertil Steril 86 (6):1561-1572

2. Jones KD, Sutton C (2002) Endometriosis. Emphasis on medical treatment is misleading. BMJ 324:115

3. Olive DL, Pritts EA (2002) The treatment of endometriosis: a review of the evidence. Ann N Y Acad Sci 955:360-372

4. Ferrero S, Abbamonte LH, Giordano M, Ragni N, Remorgida V. (2006) Deep dyspareunia and sex life after laparoscopic excision of endometriosis. Hum Reprod 22:1142-1148

5. Lyons SD, Chew SS, Thomson AJ, Lenart M, Camaris C, Vancaillie TG, Abbott JA (2006) Clinical and quality-of-life outcomes after fertility-sparing laparoscopic surgery with bowel resection for severe endometriosis. J Minim Invasive Gynecol 13(5):436-441

6. Garry R, Clayton R, Hawe J (2000). The effect of endometriosis and its radical laparoscopic excision on quality of life indicators. BJOG 107(1):44-54

7. Redwine DB, Wright JT (2001) Laparoscopic treatment of complete obliteration of the cul-de-sac associated with endometriosis: longterm follow-up of en bloc resection. Fertil Steril 76(2):358-365

8. Ford J, English J, Miles WA, Giannopoulos T (2004) Pain, quality of life and complications following the radical resection of rectovaginal endometriosis. BJOG 111(4):353-356

9. Keckstein J, Ulrich U, Kandolf O, Wiesinger H, Wustlich M (2003) Laparoscopic therapy of intestinal endometriosis and the ranking of drug treatment (in German). Zentralbl Gynakol 125(7-8):259-266

10. Vercellini P, Trespidi L, De Giorgi O, Cortesi I, Parazzini F, Crosignani PG (1996) Endometriosis and pelvic pain: relation to disease stage and localization. Fertil Steril 65(2):299-304

11. Cornillie FJ, Oosterlynck D, Lauweryns JM, Koninckx PR (1990) Deeply infiltrating pelvic endometriosis: histology and clinical significance. Fertil Steril 53:978-983

12. Gustilo-Ashby AM, Paraiso MF (2006) Treatment of urinary tract endometriosis. J Minim Invasive Gynecol 13(6):559-565

13. Schneider A, Touloupidis S, Papatsoris AG, Triantafyllidis A, Kollias A, Schweppe KW (2006) Endometriosis of the urinary tract in women of reproductive age. Int J Urol 13(7):902-904 